\title{
Preimplantation genetic diagnosis for gender selection: You don't always get what you want
}

\author{
Tanmoy Mukherjee ${ }^{1,2^{*}}$, Eric Flisser ${ }^{1}$, Alan B. Copperman ${ }^{1,2}$, Lawrence Grunfeld ${ }^{1,2}$, \\ Benjamin Sandler ${ }^{1,2}$, Jason Barritt ${ }^{1,2}$ \\ ${ }^{1}$ Reproductive Medicine Associates of New York, New York, USA \\ ${ }^{2}$ Reproductive Endocrinology, Mount Sinai School of Medicine, New York, USA \\ Email: ${ }^{\text {tmukherjee@rmany.com }}$
}

Received 16 May 2012; revised 20 June 2012; accepted 2 July 2012

\begin{abstract}
Parenting children of opposite genders is a powerful motive for parents to seek "sex-selection" services. Medical beneficence and patient autonomy support making these services available. Our goals in this study included data to permit proper patient education, assess outcome, and evaluation of potential biases in this technology. IVF/PGD cases from August 2004 to December 2009 were studied $(n=122)$. FISH was used to analyze nuclear DNA of biopsied embryos. The variables analyzed were patient age, Day 3 Fluorescent In Situ Hybridization (FISH), the number of fertilized embryos, the number of embryos biopsied, Preimplantation Genetic Diagnosis (PGD) results, the number of embryos transferred, and the fate of remaining embryos. Female embryos were sought in 84 cycles, and male embryos desired in $\mathbf{3 8}$ cycles. Couples seeking female offspring had a reduced likelihood of a female-only transfer vs. those seeking males $(p<0.001)$. No transfer was performed in 32 cases for lack of normal embryos of desired gender. Clinical pregnancy rate per embryo transfer was $30.4 \%$. PGD success rates for gender variety were lower than expected comparative to traditional IVF. In this report we present our clinical experience with IVF/PGD for gender selection. We attempt to analyze which patients seek this specialized treatment and to provide direct clinical and laboratory outcome data from our completed cycles.
\end{abstract}

Keywords: IVF; PGD; Gender Preference; Sex Selection; Family Balancing

\section{INTRODUCTION}

Application of preimplantation genetic diagnosis (PGD) during embryonic development to identify the presence

\footnotetext{
${ }^{*}$ Corresponding author.
}

of lethal genetic diseases in cycles of assisted reproduction (ART) produced the first human live births in 1990 [1]. PGD testing has since been utilized to identify a range of diseases (e.g. cystic fibrosis, sickle cell disease, hemophilia A and B, Lesch-Nyhan syndrome, Duchenne's Muscular Dystrophy), and the detection of additional genetic disorders is increasingly possible as the genetic loci of diseases are identified [2-6]. In addition to detection of single gene defects, technical advances in single-cell genetic analysis, including single nucleotide polymorphism (SNP) array, comparative genomic hybridization (CGH), and whole genome amplification (WGA), may improve diagnostic precision and permit useful preimplantation genetic screening (PGS) in patients with recurrent pregnancy loss and unexplained in vitro fertilization (IVF) treatment failure, where chromosomal errors are the result of potentially de novo mutations or meiotic and mitotic aberrations [2,7-11].

Elective sex "selection" is a controversial variant of PGD, enabling patients to identify the genetic gender of embryos prior to transfer to the uterus, thus allowing preferential selection in the absence of medical necessity. The incidence of patient interest in non-medical elective sex selection in the United States is difficult to estimate because the Society for Assisted Reproductive Technology (SART), the agency tasked with tracking the use and outcomes of in vitro fertilization (IVF) treatment in the US, does not specifically denote PGD cycles undertaken for the purposes of gender selection [8-11]. However, there is significant interest in this non-medical application. Dahl found that $8 \%$ of respondents would use preconception sex selection services [12] and Jain et al. found that $40.8 \%$ of women undergoing IVF would select the gender of their child if they could easily do so [13]. In contrast to preconception techniques for gender selection, such as sperm-sorting protocols, the issue of gender selection during cycles of assisted reproduction, and especially so in the absence of infertility or genetic disease, is additionally complicated by cost scale, alloca- 
tion of limited resources, and ethical considerations regarding the fate of supernumerary embryos, both of the preferred gender and otherwise. Alternative options, such as preconception techniques to influence embryo gender using sperm sorting protocols including gradients or centrifugation, however, lack the specificity and superior outcomes of IVF-PGD [14].

Gender variety or "family balancing" as it is sometimes known, is a particular form of PGD that is undertaken in families in which all offspring are of the same gender. Patients pursuing this option are interested in the unique experience of raising a child of the unrepresented gender $[15,16]$. The motivation of couples seeking this application of PGD in this setting may be different from that of other patients seeking gender selection, suggesting that gender variety and sex selection may be considered distinct applications of PGD/ART $[15,17]$. Increasing utilization of gender variety has prompted various ART regulatory organizations to formulate criteria for permitting application of PGD in this scenario. In 2002, the Human Fertilization and Embryology Authority (HFEA) of the United Kingdom attempted to codify the practice by limiting its application to families in which no children of a given gender existed, but conceded that a gender imbalance of more than two when both genders were represented in an existing family would also fulfill the prerequisite [18].

The ethics committees of both the American Congress of Obstetricians and Gynecologists (ACOG) and the American Society for Reproductive Medicine (ASRM) have established acceptable medical justifications for sex selection. However, the committees differ on the ethics of gender selection for non-medical indications [19]. The ACOG Ethics Committee opposes sex selection for personal and family reasons, including family balancing, because of the concern that such requests may ultimately support sexist practices. The ASRM committee recognizes the serious ethical concerns that the application of these techniques raise and counsels against their widespread use while additionally emphasizing that practitioners offering assisted reproductive services are not under legal or ethical obligation to provide non-medically indicated methods of gender selection. The committee, however, concludes that, "Until a more clearly persuasive ethical argument emerges, or there is stronger empirical evidence that most choices to select the gender of offspring would be harmful, policies to prohibit or condemn as unethical all uses of non-medically indicated preconception gender selection are not justified [20,21].” A primary concern is that when widely applied, services for gender selection can distort the natural sex ratio and lead to gross societal gender imbalance, as has occurred in China and India through the use of abortion [22-24]. In the United States, for a gross gender imbalance to oc- cur, a number of prerequisites are necessary. First, a strong preference for one gender over another must exist. And, second, child bearing must preclude conceiving a child of the opposite gender.

Couples seeking gender variety may constitute a special case of elective gender selection. The experience of parenting a child of the opposite gender is unique and may present a powerful motivator for parents whose offspring are comprised of a single gender [25-30]. As discussion of PGD utilization for gender variety evolves, not only is it essential to discuss ethical arguments in the abstract, it is also necessary to analyze empiric practice data in order to reconcile practical and theoretical aspects of this controversial therapeutic intervention. In this report we present our clinical experience with IVF/PGD for gender selection. We attempt to analyze which patients seek this specialized treatment and to provide direct clinical and laboratory outcome data from our completed cycles.

\section{MATERIALS AND METHODS}

All IVF/PGD cases for gender selection performed in our clinic from August 2004 to December 2009 were included in this study $(\mathrm{n}=122)$. All patients were counseled by their medical providers and then provided informed consent to participate in IVF/PGD. Patients underwent ovarian stimulation with gonadotropins using GnRH-antagonist or GnRH-agonist luteal-phase down regulation with or without birth control pill overlap to prevent premature luteinization of follicles. Serial monitoring by a physician was performed for controlled ovarian stimulation by hormone and ultrasound analysis. When at least two follicles measured $18 \mathrm{~mm}$ in diameter, Human Chorionic Gonadotropin (HCG) (5000 - 10,000 IU intramuscularly) or ovidrel (250 mcg subcutaneously) was administered by injection and transvaginal ultrasound-guided oocyte retrieval was performed 36 hours later. In all cases, intracytoplasmic sperm injection (ICSI) was performed. All patients had embryo biopsy performed on Day 3 after oocyte retrieval, by direct aspiration of a single blastomere through an opening created by acidified Tyrodes degradation of the zona pellucida. The biopsied blastomere was fixed to a glass microscope slide and the cytoplasm was removed before PGD analysis.

Nuclear DNA was analyzed by Fluorescent In Situ Hybridization (FISH). 2-chromosome (X, Y), 3-chromosome (21, X, Y), 5-chromosome (13, 18, 21, X, Y) or 10 -chromosome $(9,13,14,15,16,18,21,22, \mathrm{X}, \mathrm{Y})$ probes were used for FISH. PGD results were evaluated by geneticists, embryologists and the physician responseble for embryo transfer on Day 5 of embryo development. Patients were counseled about the FISH results prior to 
embryo transfer to determine the gender and number of embryos to transfer.

Variables analyzed included female patient age, day 3 FSH, basal antral follicle count (BAFC), number of retrieved oocytes, number of oocytes injected by Intracytoplasmic Sperm Injection (ICSI), number of fertilized embryos, number of embryos biopsied, PGD results, number of embryos transferred, and the fate of the remaining embryos. Patients were categorized by preferred embryo gender (female or male) and by country of residence (US or foreign). Statistical analysis was performed using Chi square test and Mann-Whitney U test for non-parametric data. A p value $<0.05$ was considered statistically significant.

\section{RESULTS}

Ninety-three patients underwent 122 IVF/PGD cycles: 69 patients attempted 1 cycle, 20 patients underwent 2 cycles, 3 patients underwent 3 cycles and 1 patient underwent 4 cycles. Female embryos were sought in 84 cycles (68.9\%) initiated, and male embryos were desired in 38 cycles $(31.1 \%)$. The distribution of FISH panels ordered were as follows: 14 (11.5\%) cases 2-probe, 16 (13.1\%) cases 3-probe, 78 (63.9\%) 5-probe and 13 (10.7\%) 10-probe. According to patient records, in 106 cycles (86.9\%), patients had at least one child prior to the IVF/PGD cycle; in 5 cases $(4.1 \%)$ no birth history was available.

Table 1 summarizes the IVF/PGD patient population by gender of interest. There were no statistical differences between the two groups in the following categories: number of days of gonadotropin administration, Day 3
Follicle Stimulating Hormone (FSH IU/l) levels, peak estradiol (E2 pg/ml), number of oocytes retrieved, and number of normally fertilized (2PN) embryos. A significant difference was noted in maternal age between those desiring females $(37.2 \pm 4.1)$ and those desiring males (34.7 \pm 4.5, $\mathrm{p}=0.003)$.

Figure 1 represents the embryo genotype according to FISH results.

A total of 919 embryos were biopsied. The average number of embryos biopsied per patient was 7.5 \pm 5.1 . A normal genotype was defined as $\mathrm{XX}$ or $\mathrm{XY}$ and the balanced presence of any autosomes analyzed. In cases where female embryos were preferred, 600 embryos underwent biopsy. The biopsy results included 249 XX embryos (41.5\%), $270 \mathrm{XY}$ embryos (45.0\%), and 81 (13.5\%) embryos with inconclusive or no result for genetic gender. For patients desiring male embryos, 319 embryos were biopsied. The biopsy results included 138 XY embryos (43.2\%), $150 \mathrm{XX}$ embryos (47.0\%), and 31 (9.7\%) embryos with inconclusive or no result. There were more male embryos in the cycles with a preference for female embryos, and more female embryos in the cycles with a preference for male embryos, although, the difference in embryo numbers did not reach statistical significance $(p=0.29)$. All embryo transfers occurred at the blastocyst stage of development on Day 5 after oocyte retrieval. The embryo transfer results from all 122 cases are shown in Figure 2.

In the 84 cases where female embryos were preferred, 58 embryo transfers were performed. Of these transfers, 51 consisted of only XX embryos, and 7 embryo transfers contained either male only or included embryos of

Table 1. IVF/PGD patient population by gender of interest.

\begin{tabular}{|c|c|c|c|c|}
\hline & Desiring female embryos $(n=84)$ & Desiring male embryos $(\mathrm{n}=38)$ & Overall $(\mathrm{n}=122)$ & $\mathrm{p}$ value \\
\hline Age & $37.2 \pm 4.1^{*}$ & $34.7 \pm 4.5^{*}$ & $36.4 \pm 4.4$ & $0.003^{*}$ \\
\hline Day 3 FSH & $7.9 \pm 2.6$ & $8.0 \pm 2.4$ & $7.9 \pm 2.5$ & 0.86 \\
\hline Peak E2 & $2232.5 \pm 1186.3$ & $2275.3 \pm 1162.6$ & $2245.3 \pm 943.9$ & 0.94 \\
\hline BAFC & $8.8 \pm 5.6$ & $9.3 \pm 6.7$ & $8.9 \pm 8.6$ & 0.71 \\
\hline Gonadotropin stimulation & $12.4 \pm 2.8$ & $12.5 \pm 3.9$ & $12.5 \pm 5.0$ & 0.98 \\
\hline Oocytes retrieved & $17.0 \pm 11.2$ & $16.8 \pm 9.1$ & $16.9 \pm 10.5$ & 0.93 \\
\hline Oocytes ICSI’d & $12.4 \pm 7.9$ & $13.7 \pm 7.8$ & $12.8 \pm 7.9$ & 0.42 \\
\hline Count of $2 \mathrm{PN}$ & $9.8 \pm 6.7$ & $10.8 \pm 6.5$ & $10.1 \pm 6.6$ & 0.44 \\
\hline Biochemical pregnancy rate & $45.2 \%$ & $47.4 \%$ & $45.9 \%$ & 0.89 \\
\hline Clinical pregnancy rate & $32.1 \%$ & $26.3 \%$ & $30.4 \%$ & 0.63 \\
\hline
\end{tabular}

${ }^{*} \mathrm{p}<0.05$ for a significant difference between those age groups desiring a male or female offspring; FSH = Follicle Stimulating Hormone (IU/l); E2 = Estradiol $(\mathrm{pg} / \mathrm{ml}) ;$ BAFC = Basal Antral Follicle Count; ICSI = Intracytoplasmic Sperm Injection; 2PN = 2 Pronuclear Embryo. 


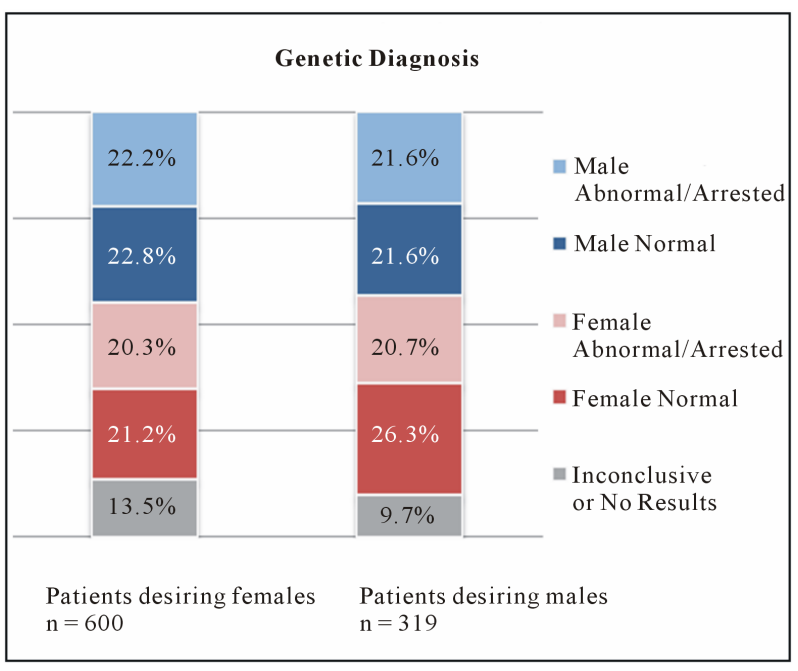

Figure 1. Embryo genotype according to FISH results. $\mathrm{n}=$ number of embryos biopsied.
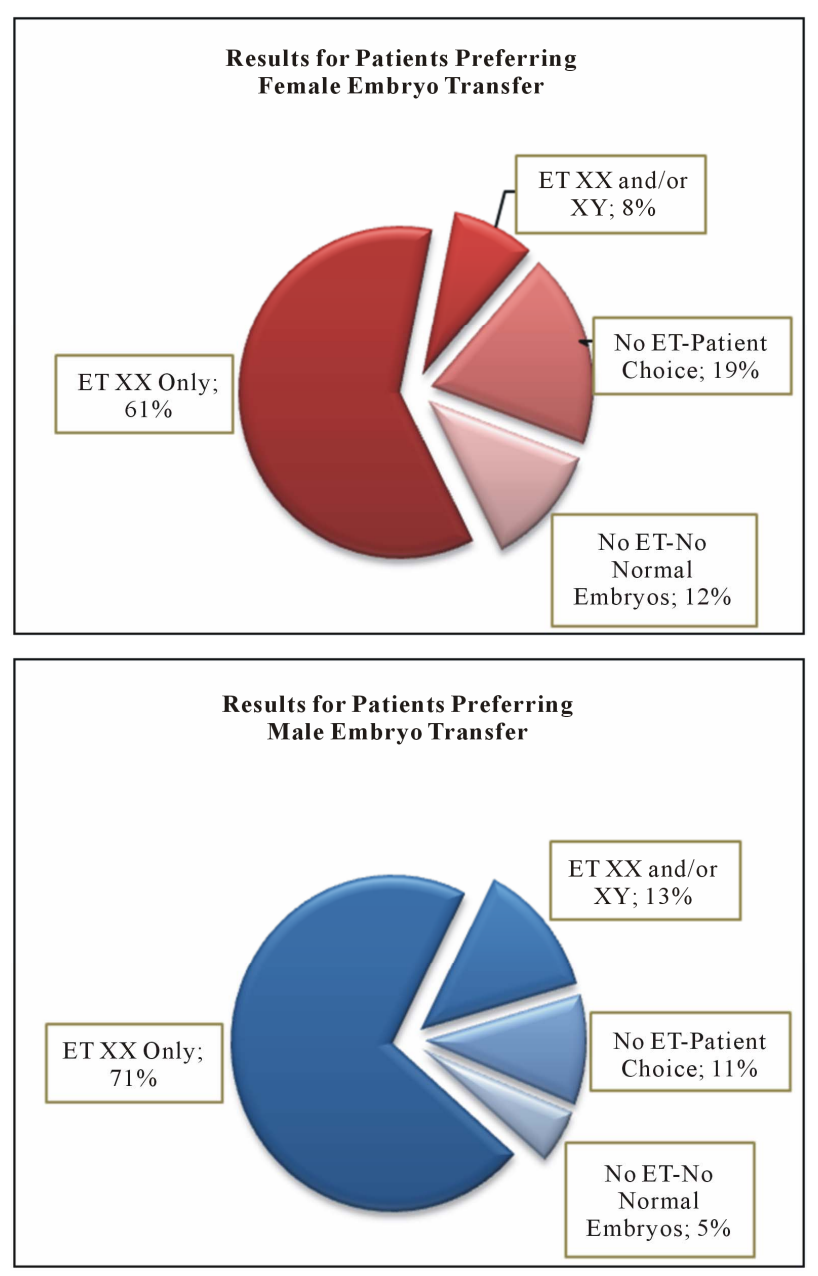

Figure 2. Embryo transfer results by gender of interest. $\mathrm{XX}=$ female, $\mathrm{XY}=$ male, $\mathrm{ET}=$ embryo transfer.

both genders. In 26 cases (31.0\%) that patients expressed a preference for female embryos but no normal XX em- bryos were available for transfer, the cycle was cancelled at the patient's request. In 38 cases requesting male embryos, 32 embryo transfers were performed. Of these transfers, 27 consisted of only XY embryos, and 5 transfers contained either female only or included embryos of both genders. In 6 cases (15.8\%) seeking male embryos for transfer where no normal XY embryos were available for transfer, the cycle was cancelled at the patient's request. Couples seeking gender variety for female offspring had a significantly reduced likelihood of a female embryo only transfer vs those seeking male offspring (p $<0.001$ )

There were 32 IVF/PGD cases (26.2\%) with cancelled embryo transfers due to the lack of normal embryos of the desired gender for transfer. In the 26 cancelled cases preferring female embryos, 10 patients had no normal embryos and 16 had viable embryos of the other gender. Two patients chose to cryopreserve supernumerary XY embryos, whereas 14 declined cryopreservation of presumed-euploid male embryos. In the 6 cancelled cases seeking male embryos without embryos for transfer, two had no normal embryos, and the remaining 4 patients with presumably-euploid XX embryos declined cryopreservation of the supernumerary embryos.

Figure 3 provides a flow diagram for the embryo transfer and cryopreservation decisions made in 122 cycles.

The biochemical pregnancy rate per embryo transfer was $45.9 \%$ (56/122). The clinical pregnancy rate (the presence of at least one intrauterine gestational sac identified by ultrasonography) per embryo transfer was $30.4 \%$ (37/122) (Table 1). For the 84 cycles with a female embryo preference, 38 had a biochemical pregnancy (45.2\%) and 27 had a clinical pregnancy (32.1\%). For the 38 cases with a male embryo preference, 18 had a biochemical pregnancy (47.4\%) and 10 had a clinical pregnancy (26.3\%).

In order to determine if a skewed preference for one gender existed based on regional preferences, the 122 IVF/PGD cycles were sorted by US residents (domestic) and non-US residents (foreign). Of 88 domestic cases, 61 were for female embryos (69.3\%) and 27 for male embryos (30.7\%). Of the 34 foreign cases, 23 were for female embryos (67.6\%) and 11 for male embryos (32.3\%). No significant difference between domestic and foreign cases was detected in terms of gender preference $(p=0.61)$.

\section{DISCUSSION}

The practice of PGD for gender variety is clinically distinct from traditional gender selection. In vitro fertilization and preimplantation genetic diagnosis is more efficacious than sperm centrifugation, medical therapy, timing of intercourse and other various interventions that 


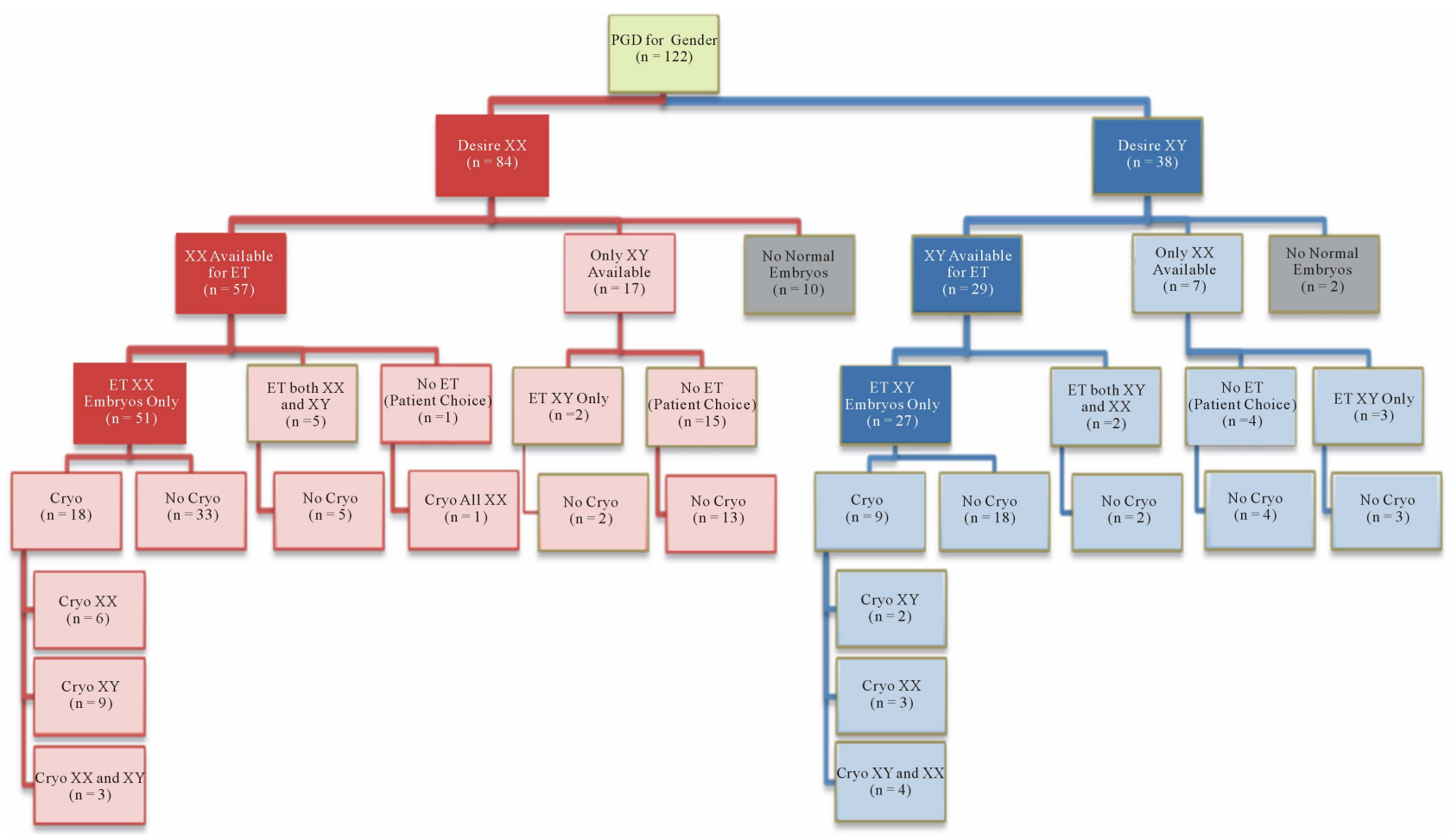

Figure 3. Gender selection patient decision tree. $\mathrm{XX}=$ female; $\mathrm{XY}=$ male; Cryo = cryopreservation; $\mathrm{PGD}=$ pre-implantation genetic diagnosis; $\mathrm{n}=$ number of patient cycles.

have been employed in efforts to alter gender proportions [31-33]. Participation in elective gender selection is potentially controversial, but its technology offers the ability to experience the unique possibility of raising a child of a specific gender. The principles of patient autonomy and medical beneficence support making gender selection services available. Nonetheless, non-malfeasance must be addressed, and proper patient counseling is mandatory for ethical application of gender variety services. In our case series, gender variety demonstrated a unique profile of gender preference, success rates, and cryopreservation utilization, which should be discussed in detail before patients undertake treatment.

Couples seeking PGD for gender variety have reduced success rates when compared to traditional IVF, there were 32 IVF/PGD cases (26.2\%) with cancelled embryo transfers due to the lack of embryos of the desired gender, abnormal genotype and/or arrested embryonic development. In total, 12 couples $(9.8 \%)$ accepted mixed gender transfers (Figure 2). While the majority of these patients had no clearly identified fertility dysfunction, the low success rate and high cancellation rate may be explained by the exclusion of approximately $50 \%$ of candidate embryos because of inconclusive results or undesired gender. Our patients are counseled that only $64 \%$ of couples have the desired embryos for transfer (Figure 2).

In our series, success rates varied with choice of gender. Couples seeking gender variety for female offspring had a reduced likelihood of having a successful transfer. One possible explanation of this observation may be a potential bias in embryo evaluation systems that appear to effectively assign a higher grade to embryos that are more likely to be genetically male [33]. This would also account for the finding that couples seeking male offspring had a greater chance to have a single gender transfer and overall a reduced cancellation rate. We also considered the possibility that families in which one gender was over represented were producing a higher than expected rate of aneuploid embryos in the unrepresented gender. Two families had extreme distributions of $\mathrm{XX}$ and $\mathrm{XY}$ embryos: in one case a couple desiring a male produced eighteen $\mathrm{XX}$ embryos and only two XY embryos, and another couple seeking female embryos produced 11 male embryos and a single female embryo. Clearly these observations, while intriguing, are not sufficient to suggest that gender imbalance is related to non-random meiotic distribution in certain families, however further data collection and analysis is certainly warranted.

In our series, twice as many couples sought to transfer female embryos than male embryos (Figure 3). Female gender preference did not vary by patient origin, suggesting that ethnographic preferences for male children were not a motivating factor in our study. It is also important to note that our patients sought treatment that was not covered by health insurance. Further assessment of 
couples seeking gender selection is necessary, but a preliminary interpretation suggests that socioeconomic status, maternal interest, and declining fertility coupled with a desire to minimize family size all play an important role in motivating couples seeking gender variety $[29,30]$.

The practice of elective gender selection frequently elicits emotional reactions concerning issues of morality and autonomy, covering a wide spectrum of opinions that include valuing all embryos as sacrosanct, libertarian attitudes towards market forces, and concerns regarding feminism and sexism. More empirical data needs to be collected before gender bias in the cases of PGD for sex selection can be definitively established. However, it must also be recognized that economic factors, such as the high cost of treatment and the requirement for sophisticated systems and technical expertise may be limiting the observation of gender bias in IVF/PGD by restricting its use from communities that lack the resources to have it performed, biasing data collection to communities where personal worth or societal status is not related to gender. In cultures where subsistence agriculture or other manual labor is the norm, prejudice in favor of male children capable of performing these tasks is a strong factor in gender bias, as it is in cultures in which male children are expected to provide continued familial fealty and support to elderly parents when they become incapable of providing for themselves. Female children may also represent additional hardship in poor communities that require a dowry for marriage. Artificial restrictions on family size, such as the "one child rule" in China, further compound imbalances [34]. In these types of communities, universal availability of PGD for gender selection would be expected to exacerbate gender imbalance [35].

However, in developed communities where distinct social gender roles have been significantly reduced, if not entirely eliminated, the preference for a single gender in offspring may not exist [36-38]. While the worldwide elective gender selection experience, whether preconceptual or antenatal, suggests a bias towards male gender, our data suggests that preference for male children is not universal. In Western families, a preference towards a balance in gender of offspring is preferred [39,40].

The application of non-medical gender selection will continue to remain a controversial topic. In our limited series, interest in elective gender selection services contradicted anticipated norms, since female embryos were more frequently sought than male embryos. The size of our sample or the community from which it was drawn may have significantly influenced our observation. In all cases, patients must be carefully counseled prior to undertaking this procedure since it is associated with a higher than anticipated failure rate related to lack of desired embryos for transfer.

\section{ACKNOWLEDGEMENTS}

Marlena Duke, MS, Lesley Chuang, MS, Casey A. McDonald, MS, Enrique Cervantes, MD, Joseph A. Lee, BS.

\section{REFERENCES}

[1] Handyside, A., Konotogianni, E.H., Hardy, K. and Winston, R.M. (1990) Pregnancies from biopsied human preimplantation embryos sexed by Y-specific DNA amplifycation. Nature, 334, 768-770. doi:10.1038/344768a0

[2] Werlin, L., Rodi, I., DeCherney, A., Marello, E., Hill, D. and Munne, S. (2003) Preimplantation genetic diagnosis (PGD) as both a therapeutic and diagnostic tool in assisted reproductive technology. Fertility and Sterility, 80, 467-468. doi:10.1016/S0015-0282(03)00605-8

[3] Harper, P.S. and Jones, L. (2002) Huntington's disease: Genetic and molecular studies. In: Bates, G., Harper, P. and Jones, L., Eds., Oxford University Press, New York, 2002, 113-158.

[4] Fiorentino, F., Biricik, A., Karadayi, H., Berkil, H., Karlikaya, G., Sertyel, S., et al. (2004) Development and clinical application of a strategy for preimplantation genetic diagnosis of single gene disorders combined with HLA matching. MHR: Basic Science of Reproductive Medicine, 10, 445-460. doi:10.1093/molehr/gah055

[5] Dreesen, J.C., Jacobs, L.J., Bras, M., et al. (2006) Multiplex PCR of polymorphic markers flanking the CFTR gene; a general approach for preimplantation genetic diagnosis of cystic fibrosis. MHR: Basic Science of Reproductive Medicine, 6, 391-396.

doi:10.1093/molehr/6.5.391

[6] Altarescu, G., Brooks, B., Margalioth, E., Eldar Geva, T., Levy-Lahad, E. and Renbaum, P. (2007) Simultaneous preimplantation genetic diagnosis for Tay-Sachs and Gaucher disease. Reproductive Biomedicine Online, 15, 8388. doi:10.1016/S1472-6483(10)60696-7

[7] The Preimplantation Genetic Diagnosis International Society PGDIS (2008) Guidelines for good practice in PGD: Programme requirements and laboratory quality assurance. Reproductive Biomedicine Online, 16, 134-147. doi:10.1016/S1472-6483(10)60567-6

[8] Wilton, L., Thornhill, A., Traeger-Synodinos, J., Sermon, K.D. and Harper, J.C. (2009) The causes of misdiagnosis and adverse outcomes in PGD. Human Reproduction, 1, $1-8$.

[9] Lewis, C.M., Pinêl, T., Whittaker, J.C. and Handyside, A.H. (2001) Controlling misdiagnosis errors in preimplantation genetic diagnosis: A comprehensive model encompassing extrinsic and intrinsic sources of error. $\mathrm{Hu}$ man Reproduction, 16, 43-50. doi:10.1093/humrep/16.1.43

[10] Piyamongkol, W., Bermúdez, M.G., Harper, J.C. and Wells, D. (2003) Detailed investigation of factors influencing amplification efficiency and allele drop-out in single cell PCR: implications for preimplantation genetic diagnosis. MHR: Basic Science of Reproductive Medicine, 9, 411420. doi:10.1093/molehr/gag051

[11] Goossens, V., Harton, G., Moutou, C., et al. (2006) Euro- 
pean Society of Human Reproduction and Embryology PGD Consortium: ESHRE PGD consortium data collection VIII: Cycles from January to December 2005 with pregnancy follow-up to October 2006. Human Reproductive, 23, 2629-2645. doi:10.1093/humrep/den238

[12] Dahl, E., Ruchi, G., Beutel, M., et al. (2006) Preconception sex selection demand and preferences in the United States. Fertility and Sterility, 85, 468-473. doi:10.1016/j.fertnstert.2005.07.1320

[13] Jain, T., Missmer, S.A., Gupta, R.S. and Hornstein, M.D. (2005) Preimplantation sex selection demand and preferences in an infertility population. Fertility and Sterility, 83, 649-658. doi:10.1016/j.fertnstert.2004.11.023

[14] Gleicher, N. and Karande, V. (2002) Gender selection for non-medical indications. Fertility and Sterility, 78, 460462. doi:10.1016/S0015-0282(02)03276-4

[15] Westoff, C.F. and Rindfuss, R.R. (1974) Sex preselection in the United States: Some implications. Science, 184, 633-636. doi:10.1126/science.184.4137.633

[16] Steinbacher, R., Gilroy, F.D. (1985) Preference for sex of child among primiparous women. Journal of Psychology: Interdisciplinary and Applied, 119, 541-547. doi:10.1080/00223980.1985.9915461

[17] Jain, T., Missmer, S.A., Gupta, R.S., Hornstein, M.D. (2005) Preimplantation sex selection demand and preferences in an infertility population. Fertility and Sterility, 83, 649-658. doi:10.1016/j.fertnstert.2004.11.023

[18] Human Fertilization and Embryology Authority (2003) Sex selection: Options for regulation. HFEA, London.

[19] ACOG Committee Opinion (2007) Sex selection. Obstetrics \& Gynecology, 109, 475-478. doi:10.1097/00006250-200702000-00063

[20] Ethics Committee of the American Society of Reproductive Medicine (1997) Sex selection and preimplantation genetic diagnosis. Fertility and Sterility, 72, 595-597.

[21] Ethics Committee of the American Society of Reproductive Medicine (2002) Preconception gender selection for nonmedical reasons. Fertility and Sterility, 75, 861-864.

[22] Plafker, T. (2002) Sex selection in China sees 117 boys born for every 100 girls. British Medical Journal, 324, 1233. doi:10.1136/bmj.324.7348.1233/a

[23] Hudson, V.M. and Den Boer, A.M. (2004) Bare branches: The security implications of Asia's surplus male population. MIT Press, Cambridge, 2004.

[24] Sen, A. (2003) Missing women-Revisited. British Medical Journal, 327, 1297-1298. doi:10.1136/bmj.327.7427.1297

[25] Davis, D. (2001) Genetic dilemmas: Reproductive technology, parental choices, and children's futures. Routledge, New York.

[26] Williamson, N.E. (1976) Sons or daughters: A crosscultural survey of parental preferences. Sage, Beverly Hills.

[27] Sloane, D.M. and Lee, C.F. (1983) Sex of previous chil- dren and intentions for further births in the United States, 1965-1976. Demography, 20, 353-367. doi:10.2307/2061247

[28] Yamaguchi, K. and Ferguson, L.R. (1995) The stopping and spacing of childbirths and their birth-history predicttors: Rational choice theory and event history analysis. American Sociological Review, 60, 272-298. doi:10.2307/2096387

[29] Pollard, M.S. and Morgan, S.P. (2002) Emerging parental gender indifference? Sex composition of children and the third birth. American Sociological Review, 67, 600-613. doi:10.2307/3088947

[30] Khatamee, M.A., Leinberger-Sica, A., Matos, P., Weseley, A.C. (1989) Sex preselection in New York City: Who chooses which sex and why. International Journal of Fertility, 34, 353-354.

[31] Fugger E.F. (1999) Clinical experience with flow cytometric separation of human $\mathrm{X}$ and $\mathrm{Y}$ Chromosome bearing sperm. Theriogenology, 52, 1435-1440. doi:10.1016/S0093-691X(99)00228-9

[32] Wilcox, A.J., Weinberg, C.R. and Baird, D.D. (1995) Timing of sexual intercourse in relation to ovulation. Effects on the probability of conception, survival of the pregnancy, and sex of the baby. New England Journal of Medicine, 333, 1517-1521. doi:10.1056/NEJM199512073332301

[33] Shettles, L.B. (1996) How to choose the sex of your baby. Main Street Books, New York.

[34] Centers for Disease Control and Prevention, American Society for Reproductive Medicine, Society for Assisted Reproductive Technology (2009) Assisted reproductive technology success rates: National summary and fertility clinic reports, Atlanta.

[35] Luna, M., Duke, M., Copperman, A.B., Grunfeld, L., Sandler, B. and Barritt, J. (2007) Blastocyst embryo transfer is associated with a sex-ratio imbalance in favor of male offspring. Fertility and Sterility, 87, 519-523. doi:10.1016/j.fertnstert.2006.06.058

[36] Allahbadia, G.N. (2002) The 50 million missing women. Journal of Assisted Reproduction and Genetics, 19, 411416. doi:10.1023/A:1016859622724

[37] Benagiano, G. and Bianchi, P. (1999) Sex preselection: An aid to couples or a threat to humanity? Human Reproduction, 14, 870-872. doi:10.1093/humrep/14.4.868

[38] Westoff, C.F. and Rindfuss, R.R. (1974) Sex preselection in the United States: Some Implications. Science, 184, 633-636. doi:10.1126/science.184.4137.633

[39] Steinbacher, R. and Gilroy, F.D. (1985) Preference for sex of child among primiparous women. Journal of Psychology: Interdisciplinary and Applied, 119, 541-547. doi:10.1080/00223980.1985.9915461

[40] Jain, T., Missmer, S.A., Gupta, R.S. and Hornstein, M.D. (2005) Preimplantation sex selection demand and preferences in an infertility population. Fertility and Sterility, 83, 649-658. 